\title{
Screening, Assessment and Management of Perioperative Malnutrition: A Survey of UK Practice
}

\section{Lewis Matthews ( $\sim$ Lewis.Matthews@uhs.nhs.uk)}

Shackleton Department of Anaesthesia University Hospital Southampton Tremona Road Southampton UK https://orcid.org/0000-0001-5967-8615

\section{Stephen Wootton}

University of Southampton

\section{Sarah Davies}

University Hospital Southampton NHS Foundation Trust

\section{Denny Levett}

University of Southampton

\section{Research}

Keywords: Nutritional risk, Preoperative evaluation, Post-operative complications, Perioperative pathways, Prehabilitation

Posted Date: October 22nd, 2020

DOI: https://doi.org/10.21203/rs.3.rs-94938/v1

License: (c) (i) This work is licensed under a Creative Commons Attribution 4.0 International License. Read Full License 


\section{Screening, assessment and management of perioperative malnutrition: A survey of UK practice}

L.S. Matthews ${ }^{1}$, S.A. Wootton ${ }^{2}$, S.J. Davies ${ }^{3}$ and D.Z.H. Levett ${ }^{4}$

${ }^{1}$ ST8 Anaesthesia and Intensive Care Medicine, University Hospital Southampton, Southampton, UK

${ }^{2}$ Associate Professor of Human Nutrition, University of Southampton, Southampton, UK

${ }^{3}$ Upper Gastro-intestinal Specialist Dietitian, University Hospital Southampton, Southampton, UK

${ }^{4}$ Professor of Perioperative Medicine and Critical Care, University Hospital Southampton,

Southampton, UK

Corresponding author:

Dr Lewis Matthews

Shackleton Department of Anaesthesia

University Hospital Southampton

Tremona Road

Southampton

UK

SO16 6YD

Lewis.Matthews@uhs.nhs.uk 


\section{Abstract}

\section{Background}

Perioperative malnutrition is common and is associated with increased mortality, complications and healthcare costs. Patients having surgery for cancer and gastro-intestinal disease are at particular risk. It is a modifiable pre-operative risk factor and perioperative clinicians are well placed to identify those at risk and instigate interventions shown to improve outcome. Thus, we conducted a survey of Perioperative Medicine Leads with the aim of assessing the current provision of nutritional screening and intervention pathways in the United Kingdom.

\section{Methods}

Perioperative Medicine Leads registered with the Royal College of Anaesthetists were asked to complete an online survey exploring current practice in screening, assessment and management of malnutrition in the perioperative period. The survey included a mixture of open and closed questions, graded response questions and options for free text. Where a response was not received departments were phoned directly and e-mails sent to non-responders.

\section{Results}

121 completed questionnaires were received from 167 Perioperative Medicine Leads (response rate of $72.5 \%)$. Seventy respondents (57.9\%) reported using the Malnutrition Universal Screening Tool to screen patients, however only $61(50.4 \%)$ referred patients at nutritional risk onto a dietitian. Sixty (49.6\%) lacked confidence in local ability to identify and manage malnutrition perioperatively, with $28(23.1 \%)$ reporting having a structured pathway for managing malnourished patients. 111 respondents (91.7\%) agreed that malnutrition impacts on quality of life after surgery and 105 (86.8\%) felt adopting a standard protocol would improve outcomes for patients. Those reporting a lack of confidence in dealing with malnutrition perioperatively cited a lack of organisational support, patients being seen too close to surgery and lack of clarity around responsibility as key reasons for difficulties in managing this group of patients.

\section{Conclusions}

Malnutrition in the perioperative period is a modifiable risk factor which is common and results in increased morbidity for patients and increased cost to healthcare systems. This survey highlights areas of practice where perioperative clinicians can improve the assessment and management of patients at nutritional risk prior to elective surgery.

\section{Trial registration}

Not applicable. 


\section{Keywords}

Nutritional risk

Preoperative evaluation

Post-operative complications

Perioperative pathways

Prehabilitation

\section{List of abbreviations}

BMI - Body Mass Index

ESPEN - European Society for Clinical Nutrition and Metabolism

MUST - Malnutrition Universal Screening Tool

NICE - National Institute for Health and Care Excellence

NRS-2002 - Nutrition Risk Screening 2002

ONS - Oral Nutritional Supplements

RCoA - Royal College of Anaesthetists 


\section{Declarations}

\section{Ethics approval and consent to participate}

We did not seek Research and Ethics Committee approval for this survey as it was voluntary, an overview of current practice, and did not involve patient contact or information.

\section{Consent for publication}

After the survey had closed respondents were contacted by the Centre for Perioperative Care to ask if there were objections to publication of the anonymised results. No objections were received.

\section{Availability of data and materials}

The survey is available in the supplementary information files. The data analysed are available from the corresponding author on reasonable request.

\section{Competing interests}

The authors declare they have no competing interests.

\section{Funding contributions}

Dr Lewis Matthews was funded by a National Institute for Health Research Academic Clinical Fellowship at the time this work was undertaken.

\section{Author's contributions}

LM conceived the project, designed the survey, and wrote the first draft of the manuscript. SD contributed to the survey design and interpretation of the data. SW and DL were major contributors in authoring the final manuscript. All authors read and approved the final manuscript.

\section{Acknowledgements}

The authors would like to thank the College Council of the Royal College of Anaesthetists and the Department of Perioperative Medicine for reviewing and disseminating the survey. 


\section{Background}

Malnutrition in the perioperative period is associated with increased morbidity, mortality, length-ofstay and healthcare costs (Weimann et al., 2020). Pre-operative malnutrition is common and is estimated to have a prevalence of up to $65 \%$ in patients undergoing surgery for cancer or gastrointestinal disease (Wischmeyer et al., 2018). These patients are at particular risk due to inadequate oral intake, cancer cachexia, muscle protein depletion and systemic inflammation (West et al., 2017; Arends et al., 2017; Bozzetti et al., 2007; Correia et al., 2001).

Optimising nutrition pre-operatively has been shown to improve outcomes after surgery (Pan et al., 2013; Vaid et al., 2012) and may impact long term health outcomes (Horowitz et al., 2015). The extent to which perioperative clinicians include screening for malnutrition and pathways for the nutritional assessment and management of patients identified as at risk is unclear. We conducted an online survey amongst Perioperative Medicine Leads to determine the current provision of nutritional screening and intervention pathways in the UK.

\section{Methods}

We did not seek Research and Ethics Committee approval for this survey as it was voluntary, an overview of current practice, and did not involve patient contact or information. After the survey had closed respondents were contacted by the Centre for Perioperative Care to ask if there were objections to publication of the anonymised results. No objections were received.

Perioperative Medicine Leads registered on the Royal College of Anaesthetists (RCOA) database in December 2018 were contacted by the Perioperative Medicine department at the RCoA by e-mail. They were asked to complete an online survey (Google Forms, Google, Mountain View, California, USA). The survey had been reviewed internally by the RCoA College Council prior to dissemination. If a response was not received departments were phoned directly and e-mails sent to non-responders on up to three occasions. Children's hospitals were excluded from the final analysis, leaving a denominator of 167 hospital trusts distributed across the UK.

Respondents were explicitly asked to discuss the survey with dietetic, nursing and surgical colleagues if necessary. We identified five areas of practice that we wanted to assess as part of the survey: i) nutritional screening, ii) assessment of malnutrition, iii) management of patients identified as malnourished or at risk of undernourishment, iv) attitudes around malnutrition in the perioperative period and v) local barriers to implementing care.

The survey included a mixture of open and closed questions, graded response questions and options for free text. Respondents were also asked to use free-text comments to identify areas of good local practice. (Supplementary information).

The survey data was exported from Google Forms (Google, Mountain View, California, USA) to Microsoft Excel (Microsoft Corporation, Redmond, Washington, USA) for further analysis.

\section{$\underline{\text { Results }}$}

We received 121 completed questionnaires from 167 Perioperative Medicine Leads in hospital trusts across the UK (response rate of 72.5\%) between December 2018 and July 2019.

\subsection{Nutritional screening}


Over $75 \%$ of respondents $(94,77.7 \%)$ indicated that pre-operative malnutrition screening was performed by nursing staff in their hospital. Other groups of healthcare professionals reported as performing screening were surgeons $(26,21.5 \%)$, anaesthetists $(25,20.7 \%)$ and dietitians $(23$, $19.0 \%)$. Fifteen respondents (12.4\%) did not know who was responsible for screening, four (3.3\%) stated that no one was responsible for screening and one respondent reported that a junior doctor was dedicated to collecting this information in their hospital.

The most widely reported approaches to identifying patients at risk of undernourishment were Body Mass Index (BMI) (75, 62.0\%), the Malnutrition Universal Screening Tool (MUST) (70, 57.9\%) and percentage weight loss (24, 19.8\%). Other tools used included the Malnutrition Screening Tool (2, 1.7\%), Short Nutritional Assessment Questionnaire $(2,1.7 \%)$ and Nutritional Risk Screening 2002 (NRS-2002) (1, 0.8\%). Fourteen respondents (11.6\%) did not know which screening tool was being used, two (1.7\%) used "disease-specific" tools and three (2.5\%) reported using no screening tool.

\subsection{Assessment of malnutrition in patients identified at risk by screening}

The commonest reported anthropometric measure was BMI $(104,86.0 \%)$. Alternative anthropometric assessments included arm muscle circumference (4,3.3\%), skin-fold thickness (2, $1.7 \%)$ and hand-grip strength $(1,0.8 \%)$. Ten respondents $(8.3 \%)$ stated that no anthropometric testing was undertaken and $10(8.3 \%)$ did not know what local practice was.

Over a third of respondents $(42,34.7 \%)$ reported that no specific biochemical assessments were performed and 11 (9.1\%) did not know what local practice was. One respondent stated that the use of biochemical assessment was "surgery dependent" and one used a "specific malnutrition screen".

As a marker of disease severity and inflammation serum albumin was the most common biochemical assessment, reported by 74 respondents (61.2\%). Other biochemical tests included total protein ( 25 , $20.7 \%), \operatorname{CRP}(15,12.4 \%)$, transferrin $(11,9.1 \%)$ and lipid studies $(8,6.6 \%)$.

An assessment of body composition, such as bioelectrical impedance analysis or CT analysis, was only performed in two hospitals (1.7\%). Ninety respondents $(74.4 \%)$ stated that no body composition assessment was undertaken and 29 (24.0\%) did not know what local practice was. One respondent stated that this was "patient dependent".

Functional testing was undertaken by 57 respondents (47.1\%), most frequently using a self-reported questionnaire $(29,24.0 \%)$, timed get-up-and-go $(16,13.2 \%)$ or stair climb $(14,11.6 \%)$. Four trusts (3.3\%) assessed functional status using cardio-pulmonary exercise testing. Forty-nine respondents (40.5\%) stated that no functional testing was undertaken and 15 (12.4\%) did not know what local practice was.

\subsection{Management of malnourished patients}

Half of the respondents (50.4\%) reported that their hospital had no perioperative pathway for managing malnourished patients. Twenty-eight respondents (23.1\%) reported that their hospital did include a perioperative pathway for malnourished patients and $17(14.1 \%)$ reported that their hospital was in the process of developing a pathway; 15 (12.4\%) did not know whether or not a formal pathway existed.

Where a patient was identified as being malnourished pre-operatively 61 respondents (50.4\%) reported that the patient would be referred to a dietitian; $12(9.9 \%)$ referred back to the surgical team, $11(9.1 \%)$ referred to the patients general practitioner, eight $(6.6 \%)$ were managed by the anaesthetic team, two $(1.7 \%)$ referred on to a gastroenterologist and two $(1.7 \%)$ had a dedicated 
perioperative nutrition team that managed the patient. Eight (6.6\%) did not know what local practice was.

Nearly half of respondents $47(38.8 \%)$ reported that oral nutritional supplements were prescribed to malnourished patients in their hospital; $46(38.0 \%)$ did not and $28(23.1 \%)$ respondents were unsure of local practice.

\subsection{Attitudes around perioperative malnutrition}

Nearly all of the respondents $(111,91.7 \%)$ either "agreed" or "strongly agreed" that malnutrition had an impact on quality of life following surgery; eight (6.6\%) "strongly disagreed" and two (1.7\%) neither "agreed" nor "disagreed".

The majority of respondents $(103,85.1 \%)$ either "agreed" or "strongly agreed" that perioperative clinicians had a role in the identification and management of malnutrition in the pre-operative period; eight (6.6\%) either "disagreed" or "strongly disagreed" and 10 (8.3\%) neither "agreed" nor "disagreed".

Approximately half of the respondents $(60,49.6 \%)$ either "disagreed" or "strongly disagreed" with the statement that "they were confident their hospital was able to identify and manage patients with malnutrition perioperatively"; 37 (30.6\%) neither "agreed" nor "disagreed" and 24 (19.8\%) either "agreed" or "strongly agreed".

The majority of respondents $(105,86.8 \%)$ either "agreed" or "strongly agreed" that adopting a standard protocol for managing perioperative malnutrition would improve patient outcomes. Three "strongly disagreed" (2.5\%) and 13 (10.7\%) neither "agreed" nor "disagreed".

\subsection{Barriers to care}

Those respondents who either "disagreed" or "strongly disagreed" with the statement that "they were confident their hospital was able to identify and manage patients with malnutrition perioperatively" ( $n=60$ ) were asked to detail their reasons for their disagreement. The main reasons given were: i) lack of organisational support $(58,96.7 \%)$, ii) patients seen too close to surgery (55, $91.7 \%)$, iii) lack of clarity around responsibility $(55,91.7 \%)$, iv) lack of training and education (38, $63.3 \%)$ and $v$ ) time constraints (32, 53.3\%). 


\section{$\underline{\text { Discussion }}$}

This is the first survey of Perioperative Medicine Leads in the UK to evaluate the structured screening, assessment and management of malnutrition in patients undergoing elective surgery. The response rate of $72.5 \%$ suggests the survey is sufficiently representative of current practice.

Perioperative medicine is defined as the patient-centred, multidisciplinary and integrated medical care from the contemplation of surgery until full recovery. Consequently, an important part of the Perioperative Medicine Lead role should be to evaluate the pathways supporting nutritional screening and optimisation pre-operatively. Malnutrition is a modifiable risk factor and all patients' nutritional status should be optimised before elective surgery (Weimann et al., 2020; Lobo et al., 2020).

We found that whilst the majority of Perioperative Medicine Leads indicated that patients were routinely screened for malnutrition in their hospital, nearly half lacked confidence that their trust had an effective process for ensuring that all surgical patients were screened and treated in the perioperative period. The majority of hospitals are reported to be using the Malnutrition Universal Screening Tool (MUST) for screening patients, however only half are reported to refer patients identified as being at nutritional risk onto a dietitian and less than a quarter have a structured pathway for managing malnourished patients pre-operatively.

The Perioperative Medicine Leads in this survey overwhelmingly agreed that malnutrition impacts on a patient's quality of life after surgery, that structured pathways for managing malnourished patients pre-operatively would improve patient outcomes and that perioperative clinicians have a role in its management. However, those that lacked confidence in their hospital's ability to identify and manage malnutrition in the surgical patient cited a lack of organisational support, proximity of seeing patients to surgery, lack of clarity around responsibility and inadequate training and education as reasons they felt they could not manage this group. So how do we bridge this gap between what we think we should be doing and reality?

\section{Screening for malnutrition}

All patients having surgery, whether elective or emergency, should be screened for nutritional risk (National Institute for Health and Care Excellence, 2017). It is the key first step in identifying those that may need additional support and is essential to avoid missing the many patients who are malnourished without displaying overt symptoms. It is therefore surprising that only two thirds of respondents stated the use of a screening tool validated for surgical patients. When a validated tool was used this was almost exclusively the MUST. Whilst MUST is recommended by the National Institute for Health and Care Excellence (NICE) for screening hospital and community patients numerous other screening tools are available. For example, the Nutrition Risk Screening 2002 (NRS2002 ) is recommended by European Society for Clinical Nutrition and Metabolism (ESPEN) in this group of patients (Weimann et al., 2020; Kondrup et al., 2003), yet only one respondent reported its use. Other examples include the Malnutrition Screening Tool, Short Nutritional Assessment Questionnaire and the recently developed Pre-operative Nutrition Score, which is a modification of MUST that also incorporates albumin (West et al., 2017). Regardless of which tool is being used it is clear from the survey that the nutritional needs of some patients are not being appropriately addressed pre-operatively and it is likely that many patients are at risk of malnutrition at the time of surgery. We would recommend that pre-operative pathways are mapped as a priority to identify the point of contemplation of surgery and that nutritional screening is performed as early as possible (Grocott et al., 2017). The screening tool used should be able to detect the presence of under- 
nutrition in an elective surgical population and should be standardised across all specialities to enable effective institution wide consistency of practice.

\section{Assessment of the patient at risk of malnutrition}

Any patient identified at being at risk of malnutrition should subsequently undergo a diagnostic assessment involving the identification of phenotypic (non-volitional weight loss, low BMI, low muscle mass) and aetiological (reduced intake, disease burden/inflammation) criteria (Cederholm et al., 2019). Whilst some of these criteria are included in many screening tools (e.g. BMI and weight loss within MUST) it is crucial to highlight that screening and assessment are temporally different processes and confusing the two may result in misdiagnosis and incorrect treatment. With specific regard to muscle mass, virtually no anthropometric or body composition assessments appear to be being performed in patients identified at nutritional risk. This omission is important as patients with higher pre-operative lean body mass cope better with surgery, have fewer complications and spend less time in hospital (Kyle et al., 2005; Pichard et al., 2004; Van Venrooij et al., 2012).

Disease burden and inflammation are harder to objectively define. In the absence of a better test hypo-albuminaemia (albumin $<30 \mathrm{~g} / \mathrm{l}$ without hepatic or renal dysfunction) may be the best biochemical marker currently available and was used by nearly two thirds of those surveyed. It is important the perioperative clinician is aware that albumin reflects disease severity and related catabolism, and is not a direct measure of malnutrition. However, it is prognostic for post-operative complications and therefore recommended by ESPEN for use in patients having surgery (Weimann et al., 2020).

The assessment of patients identified as being at nutritional risk should ideally be undertaken by those with experience and accredited professional training in nutrition, such as a registered dietitian or physician with specific responsibility for clinical nutrition. However, current dietetic resources are largely directed to supporting patients after surgery. Outside of specialities where there is a very high risk of malnutrition, such as upper gastro-intestinal cancer, pathways for pre-operative optimisation may be under-resourced and lack specialist dietitian input. It was, therefore, unsurprising that only half of those identified to be at risk received onward referral to a dietitian, with the remainder seeing a mixture of professions including surgeons, anaesthetists, general practitioners or in some instances no one at all. The reason for this variability will be multi-factorial and may reflect an under-resourced pathway that is not highly structured. In keeping with this, we report that less than half of Perioperative Medicine Leads reported having a pathway for managing malnourished patients despite the vast majority agreeing this would improve patient outcomes. One solution to better direct the use of available resources may lie in formalising pathways and reengineering the patient's perioperative journey such that those at risk are identified at the time of referral for surgery, assessed earlier, with sufficient time then afforded to the interventions required to improve modifiable risk factors such as malnutrition (Grocott et al., 2017).

\section{Management of malnourished patients pre-operatively}

The aims of treatment of a malnourished surgical patient are to improve nutritional status, limit wasting and ultimately maximise resilience and functional recovery. Where this cannot be achieved by dietary advice and food alone nutritional support may be required (oral nutritional supplements (ONS), enteral tube feeding and/or parenteral nutrition). We did not examine enteral tube feeding or parenteral nutrition but did explore ONS as this is a simple intervention that can be actioned preoperatively. NICE recommends considering ONS in those who are malnourished or at risk of malnutrition (National Institute for Health and Care Excellence, 2017) as ONS has consistently been shown to increase caloric intake (Sobotka, 2010), reduce post-operative complications (Waitzberg et 
al., 2006) and be cost-effective (Elia et al., 2016). ESPEN goes further in recently published recommendations, suggesting it be obligatory for all malnourished cancer and high-risk surgical patients and patients not receiving adequate intake through normal food (Lobo et al., 2020). Given this we were surprised that less than half of the Perioperative Medicine Leads report that their hospital prescribe ONS for malnourished patients. When considering the implementation of ONS into a nutritional care plan it should be noted that patients need to be educated about its benefits and consideration given to the provision of energy-dense $(>2 \mathrm{kcal} / \mathrm{ml})$ formulations as both improve compliance (Grass et al., 2015). The benefit of pre-operative immune-enriched ONS, so called "immuno-nutrition", over a diet supplemented with iso-nitrogenous "standard" ONS remains unproven (Hegazi et al., 2014).

So how can we improve the care we provide for this group of patients? Screening, assessing and managing these patients is important because malnutrition is one of the few modifiable preoperative risk factors that, if addressed early and treated appropriately, can have an impact on postoperative outcomes (Stratton and Elia, 2007; Jie et al., 2012; Garth et al., 2010). Malnutrition is under-recognised and under-treated resulting in immune dysfunction, poor wound healing, muscle weakness and impaired fluid homeostasis (British Association of Parenteral and Enteral Nutrition, 2009; Marcos et al., 2003). It causes increased postoperative morbidity, excess mortality and increased costs (Weimann et al., 2020; Schneider et al., 2004; Sorensen et al., 2008). Cancer patients are at particular risk due to the effects of malignancy on nutrient metabolism and delays in surgery due to the side-effects of neo-adjuvant treatments (Andreyev et al., 1998). As a case in point a recent study of patients undergoing colorectal cancer surgery found that patients were more likely to be readmitted within 30 days if malnourished (Gillis et al., 2015).

There are numerous issues highlighted by this survey, some easier to remedy than others. Screening is a relatively simple intervention and we would encourage all Perioperative Medicine Leads to introduce a standardised screening tool early in the surgical pathway as a priority, as the first stage of identifying the size of this unmet need. Ideally all patients identified as at risk should be assessed by a dietitian, although there may be significant resource implications if hospital dietitians are primarily focused on in-hospital patients. Early identification is essential to ensure there is sufficient time for meaningful optimisation without delaying surgery. Involving dietitians in the development of standardised pre-operative pathways is key and will facilitate the multidisciplinary teamwork and data collection that is required to ensure an adequately resourced service. Important questions include: who should be prioritised for dietitian assessment; should all patients should be given ONS if the time before surgery is limited and dietitians may not be available to assess all patients immediately; how can pre-operative care be integrated with community dietitian services?

Looking to the future, technology should be embraced as a way of improving the way we manage malnourished surgical patients. It is already possible for muscle mass to be assessed using bioelectrical impedance analysis or cross-sectional imaging, for nutrition risk scores to be transferred seamlessly between primary and secondary care and for patients to take responsibility for their own nutritional health through the use of applications on smart devices. Nutrition therapy is also a key component of multi-modal prehabilitation, recently advocated for all people with cancer, alongside psychological and exercise interventions (Macmillan Cancer Support, 2019). Nutritional prehabilitation alone or combined with exercise reduces length of stay by two days in colorectal cancer surgery patients and may result in faster return to pre-operative fitness (Gillis et al., 2018). The results of larger randomised controlled trials are awaited.

One of the main strengths of this study is the high response rate, implying it is representative of current practice. Additionally, we focused on the areas of nutritional evaluation that are clearly within the domain of Perioperative Medicine Leads, namely standardised screening, pathway 
management and barriers to care rather than focusing on specific dietetic expertise i.e. method of nutritional assessment, decisions regarding enteral tube feeding or parenteral nutrition. As such our findings are most relevant to the perioperative clinician seeing patients prior to elective surgery in the pre-assessment clinic. However, our study has weaknesses. We relied on Perioperative Medicine Leads being able to discuss questions with other members of the multi-disciplinary team if there was uncertainty. Furthermore, whilst we believe that Perioperative Medicine Leads should be aware of local polices and nutritional services available in their hospital this may not always be the case.

In conclusion we report that there are deficiencies in the screening, assessment and optimisation of nutritional status prior to elective surgery in the United Kingdom. There is an urgent need to implement standardised pathways to ensure the optimisation of a risk factor that we know is amenable to intervention in a realistic pre-operative time frame and that has important impact on surgical outcomes. 


\section{Acknowledgements}

The authors would like to thank the College Council and the Department of Perioperative Medicine at The Royal College of Anaesthetists for reviewing and disseminating the survey. 


\section{References}

1. Andreyev HJN, Norman AR, Oates J, Cunningham D. Why do patients with weight loss have a worse outcome when undergoing chemotherapy for gastrointestinal malignancies? European Journal of Cancer. 1998;34:503-509.

2. Arends J, Bachmann $P$, Baracos $V$ et al. ESPEN guidelines on nutrition in cancer patients. Clinical Nutrition. 2017;36:11-48.

3. Bozzetti F, Gianotti L, Braga M, Di Carlo V, Mariani L. Postoperative complications in gastrointestinal cancer patients: The joint role of the nutritional status and the nutritional support. Clinical Nutrition. 2007;26:698-709.

4. British Association of Parenteral and Enteral Nutrition. Combating malnutrition: recommendations for action. Report from the Advisory Group on malnutrition. 2009. https://www.bapen.org.uk/pdfs/reports/advisory_group_report.pdf. Accessed 2nd September 2020.

5. Cederholm T, Jensen GL, Correia MITD et al. GLIM criteria for the diagnosis of malnutrition - A consensus report from the global clinical nutrition community. Journal of Cachexia, Sarcopenia and Muscle. 2019;10:207-217.

6. Correia MITD, Caiaffa WT, Da Silva AL, Waitzberg DL. Risk factors for malnutrition in patients undergoing gastroenterologicl and hernia surgery: An analysis of 374 patients. Nutricion Hospitalaria. 2001;16:59-65.

7. Elia M, Normand C, Norman K, Laviano A. A systematic review of the cost and cost effectiveness of using standard oral nutritional supplements in the hospital setting. Clinical Nutrition. 2016;35:370-380.

8. Garth AK, Newsome CM, Simmance N, Crowe TC. Nutritional status, nutrition practices and postoperative complications in patients with gastrointestinal cancer. Journal of Human Nutrition and Dietetics. 2010;23:393-401.

9. Gillis C, Buhler K, Bresee L et al. Effects of Nutritional Prehabilitation, With and Without Exercise, on Outcomes of Patients Who Undergo Colorectal Surgery: A Systematic Review and Metaanalysis. Gastroenterology. 2018;155:391-410.

10. Gillis $C$, Nguyen TH, Liberman AS, Carli F. Nutrition adequacy in enhanced recovery after surgery: A single academic center experience. Nutrition in Clinical Practice 2015;30:414-419.

11. Grass F, Bertrand PC, Schäfer M et al. Compliance with preoperative oral nutritional supplements in patients at nutritional risk only a question of will? European Journal of Clinical Nutrition. 2015;65:525-529.

12. Grocott MPW, Plumb JOM, Edwards M, Fecher-Jones I, Levett DZH. Re-designing the pathway to surgery: better care and added value. Perioperative Medicine. 2017;6:9.

13. Hegazi RA, Hustead DS, Evans DC. Preoperative standard oral nutrition supplements vs immunonutrition: Results of a systematic review and meta-analysis. Journal of the American College of Surgeons. 2014;219:1078-1087.

14. Horowitz M, Neeman E, Sharon E, Ben-Eliyahu S. Exploiting the critical perioperative period to improve long-term cancer outcomes. Nature Reviews Clinical Oncology. 2015;12:213-226.

15. Jie B, Jiang ZM, Nolan MT, Zhu SN, Yu K, Kondrup J. Impact of preoperative nutritional support on clinical outcome in abdominal surgical patients at nutritional risk. Nutrition. 2012;28:10221027.

16. Kondrup J, Allison SP, Elia M, Vellas B, Plauth M. ESPEN guidelines for nutrition screening 2002. Clinical Nutrition. 2003;22:415-21.

17. Kyle UG, Pirlich M, Lochs H, Schuetz T, Pichard C. Increased length of hospital stay in underweight and overweight patients at hospital admission: A controlled population study. Clinical Nutrition. 2005;24:133-142.

18. Lobo DN, Gianotti L, Adiamah A, et al. Perioperative nutrition: Recommendations from the ESPEN expert group. Clinical Nutrition. 2020. doi:10.1016/j.clnu.2020.03.038. 
19. Macmillan Cancer Support. Prehabilitation for People with Cancer. 2019.

https://www.macmillan.org.uk/_images/prehabilitation-guidance-for-people-with-cancer_tcm9353994.pdf. Accessed $16^{\text {th }}$ August 2020.

20. Marcos A, Nova E, Montero A. Changes in the immune system are conditioned by nutrition. European Journal of Clinical Nutrition. 2003;57:S66-S69.

21. National Institute for Health and Care Excellence. Nutrition support for adults: oral nutrition support, enteral tube feeding and parenteral nutrition (CG32). 2017.

https://www.nice.org.uk/Guidance/Cg32. Accessed 2nd September 2020.

22. Pan $\mathrm{H}$, Cai S, Ji J, Jiang Z, Liang H, Lin F, Liu X. The impact of nutritional status, nutritional risk, and nutritional treatment on clinical outcome of 2248 hospitalized cancer patients: a multicenter, prospective cohort study in Chinese teaching hospitals. Nutrition and Cancer. 2013;65:62-70.

23. Pichard C, Kyle UG, Morabia A, Perrier A, Vermeulen B, Unger P. Nutritional assessment: Lean body mass depletion at hospital admission is associated with an increased length of stay. American Journal of Clinical Nutrition. 2004;79:613-618.

24. Schneider SM, Veyres $P$, Pivot $X$ et al. Malnutrition is an independent factor associated with nosocomial infections. British Journal of Nutrition. 2004;92:105-111.

25. Sobotka L. Basics in Clinical Nutrition. $4^{\text {th }}$ ed. Czech Republic: Galen; 2010.

26. Sorensen J, Kondrup J, Prokopowicz J et al. EuroOOPS: An international, multicentre study to implement nutritional risk screening and evaluate clinical outcome. Clinical Nutrition. 2008;27: 340-349.

27. Stratton RJ, Elia M. Who benefits from nutritional support: What is the evidence? European Journal of Gastroenterology and Hepatology. 2007;19:353-358.

28. Vaid S, Bell T, Grim R, Ahuja V. Predicting risk of death in general surgery patients on the basis of preoperative variables using American College of Surgeons National Surgical Quality Improvement Program data. The Permanente journal. 2012;16:10-17.

29. Van Venrooij LMW, Verberne HJ, de Vos R, Borgmeijer-Hoelen MMMJ, van Leeuwen PAM, de Mol BAJM. Postoperative loss of skeletal muscle mass, complications and quality of life in patients undergoing cardiac surgery. Nutrition. 2012;28:40-45.

30. Waitzberg DL, Saito H, Plank LD et al. Postsurgical infections are reduced with specialized nutrition support. World Journal of Surgery. 2006;30:1592-1604.

31. Weimann A, Braga M, Carli F et al. ESPEN guideline: Clinical nutrition in surgery. Clinical Nutrition. 2020;36:623-650.

32. West MA, Wischmeyer PE, Grocott MPW. Prehabilitation and Nutritional Support to Improve Perioperative Outcomes. Current Anesthesiology Reports. 2017;7:340-349.

33. Wischmeyer PE, Carli F, Evans DC et al. American Society for Enhanced recovery and perioperative quality initiative joint consensus statement on nutrition screening and therapy within a surgical enhanced recovery pathway. Anesthesia and Analgesia. 2018;126:1883-1895. 


\section{Supplementary Files}

This is a list of supplementary files associated with this preprint. Click to download.

- Supplementarymaterial1.pdf 